\title{
Analysis and Comparison of Modified LAR1 (MYLAR1) Routing Protocol with LAR1, OLSR INRIA, DSR, ZRP, and OSPF v2
}

\author{
Aakash Jasper \\ M.Tech Scholar \\ ECE Department, SSET \\ SHIATS-DU \\ Allahabad, U.P., India
}

\author{
Rajeev Paulus \\ Assistant Professor \\ ECE Department, SSET \\ SHIATS-DU \\ Allahabad, U.P., India
}

\author{
A.K. Jaiswal \\ Professor, H.O.D, \\ ECE Department, SSET \\ SHIATS-DU \\ Allahabad, U.P., India
}

\begin{abstract}
In our previous work we created a new ad hoc routing protocol MYLAR1, by modifying LAR1 routing protocol. The previous work showed that MYLAR1 routing protocol showed better performance as compared to LAR1 routing protocol in performance parameters such as: end to end delay, throughput, jitter and packets received without error. This paper discusses the comparison of MYLAR1 with other ad hoc routing protocols such as OLSR INRIA, DSR, ZRP, OSPF v2 and original LAR1 routing protocol. The results show that MYLAR1 routing protocol performs better as compared to other ad hoc routing protocols. QualNet 6.1 Network Simulator is used to simulate the proposed work.
\end{abstract}

\section{Keywords}

MYLAR1, LAR1, OLSR INRIA, DSR, ZRP, OSPF v2

\section{INTRODUCTION}

In recent times communication is moving from wired to wireless. There has been a boom in the ad hoc wireless communication systems which do not require infrastructure like cellular communication. MANET (Mobile ad hoc network) is an example of a communication system which has the capability to self organise and its nodes (or workstations) communicate via multi hop [1]. Since the component nodes of a MANET are mobile, the network continuously changes dynamically. A number of ad hoc routing protocols have been developed for a successful and efficient communication between different nodes in an ad hoc network. There are three categories of ad hoc routing protocols: flat routing, hierarchical routing and geographical position assisted routing [2]. Flat routing can be further sub-divided into two categories: pro active (table driven) routing and reactive (on demand) routing. In flat routing scheme, equal role is played by each node and scheme of flat addressing is utilised. Proactive routing involves exchange of background routing information by all the nodes in the network. Examples of proactive routing are FSR, FSLS, OLSR, TBRPF etc. Reactive routing is very popular in wireless ad hoc routing scheme category. The basic concept of reactive routing is that all the nodes in the wireless ad hoc network try to reduce the routing overhead by sending routing packets only when communication between any two or more nodes is to take place. Examples of reactive routing are AODV, DSR etc. Hierarchical routing involves nodes in the network organised in groups and each node different functions outside and inside the group. The update packet size and the routing table size of component nodes of the network decreases significantly by including the nodes in only to a part of the network rather than the whole network. This reduces the control overhead in wireless ad hoc hierarchical routing. Examples of hierarchical routing are HSR, CGSR, ZRP, LANMAR etc. Geographic position assisted routing involves transfer of messages using geographical position and other physical parameters (such as velocity etc.) of nodes rather than the logical position only. Examples of geographic position assisted routing are GeoCast, LAR, DREAM, GPSR etc. In this work our modified LAR1 routing protocol (MYLAR1) from our previous work [4] had been analysed and compared with the ad hoc routing protocols from each of the above told categories. MYLAR1 is compared with OLSR INRIA (a pro active routing protocol), DSR (a reactive routing protocol), ZRP (a hierarchical routing protocol) and the original LAR1 itself (a geographical position assisted routing protocol). OSPF v2 is also compared with MYLAR1 and other ad routing protocols. QualNet 6.1 is used in this work to compare the ad hoc routing protocols. QualNet 6.1 is a network simulator that mimics the behaviour of a real network [12].

\section{WIRELESS AD HOC ROUTING PROTOCOLS}

\subsection{MYLAR1}

MYLAR1 is the modification of original LAR1 routing protocol which was done in our previous work [4] and resulted in improvement of the original LAR1 routing protocol. In this routing protocol rather than using original three types of routing packets: route request, route reply and route error packets, four types of routing packets: route request, modified route request, route reply and route error packets are used. When communication is to take place modified route request packets are used. The original route request packets are used when the path for data flow is broken. The modified route request packet is different because redundant fields like flooding variable and zone variable are absent in its frame format. Even in the original route request packet flooding variable is removed. Due to the absent fields in the route request frame format the overload of the network decreases increasing the overall efficiency of the network. To avoid too much flooding of routing packets only those nodes where RSS $>-85 \mathrm{dBm}$ the routing packets will be send.

\subsection{LAR1}

LAR1 (Location Aided Routing scheme 1) is a reactive, position and velocity based routing protocol. The nodes in the network use GPS (Global Positioning System) location of the nodes to transfer data from one place in the network to another. If a node in the network wants to communicate with another node in the network first a wireless communication link needs to be established. LAR1 uses route request, route reply and route error packets to establish and re-establish (when the communication link breaks due to some error) communication link. First the source node calculates the expected zone which is the area where the destination node could be found. Expected zone is a circular area calculated on the basis of previous location and average velocity of the 
destination node. Based on the expected zone a smallest possible rectangle is estimated containing the expected zone circle and the source node with farthest most corner of the rectangle as shown in Fig. 1. The rectangular area is called request zone. The route request packets are flooded only in the request zone, if a node outside the request zone receives a route request packet it will simply drop the route request packet. For example as shown in Fig. 1 if node $\mathrm{M}$ receives the route request packet from source $\mathrm{S}$ it will process the packet further since it lies inside the request zone, however if node $\mathrm{N}$ receives the route request packet it will simply drop the packet.

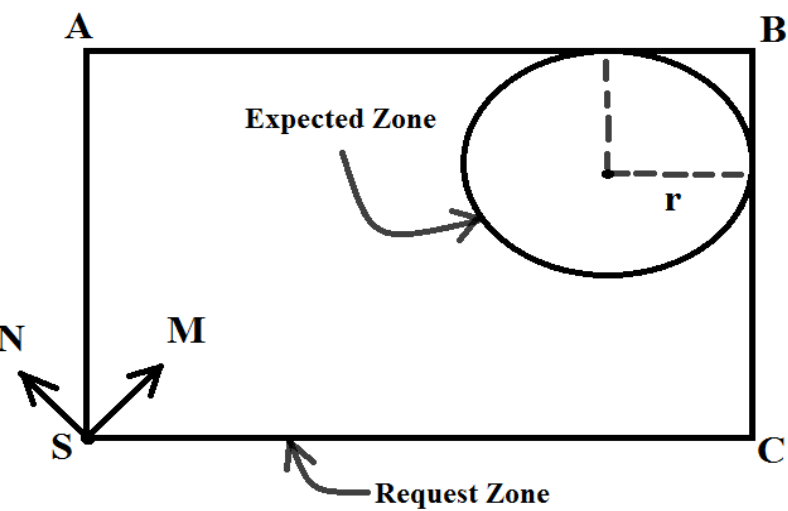

Fig 1: LAR scheme 1

\subsection{OLSR INRIA}

OLSR (Optimised Link State Routing) was developed by French National Institute for Research in Computer Science and Control (INRIA). OLSR was created for mobile ad hoc networks. OLSR functions in a pro active and table driven manner; hence topology information is exchanged between all the nodes on a periodic basis. The primary goal of this protocol is to minimize as far as possible the control traffic. To do this the tropical information is flooded by only a group of small number of nodes called Multi Point Relays (MPR). MPRs are used when an optimal route needs to be calculated from source to destination. OLSR is well suited for large and dense network.

\subsection{DSR}

DSR (Dynamic Source Routing) is a reactive or on demand routing protocol. It is designed mainly for use in wireless ad hoc multi hop networks of mobile nodes. It is quite similar to LAR1. It builds the route on demand when a node in the network wants to communicate by simply flooding the route request packets without the prior knowledge of any known route. DSR may use a set of optimizations to route the packets more efficiently and reduce the control overheads.

\subsection{ZRP}

ZRP (Zone Routing Protocol) is a hierarchical ad hoc routing protocol. It is a hybrid routing protocol which divides the network into overlapping zones. It runs independent protocols between and within the zones created. ZRP uses IERP (Inter zone Routing Protocol) for inter zone routing; IARP (Intra zone Routing Protocol) for intra zone routing and BRP (Bordercast Resolution Protocol) is used to optimize the routing process between the perimeter nodes. IARP is a pro active routing protocol used inside a zone. It can also be used as a standalone mode. IERP is used to discover a route to remote nodes outside the zone of the node. It can also be used as a standalone routing protocol. It is an on demand or reactive routing protocol. BRP is used to efficiently flood broadcast packets throughout the network. It is not a full featured routing protocol.

\subsection{OSPF v2}

OSPF (Open Shortest Path First) is a link state routing protocol. OSPF is defined as OSPF v2 in RFC 2327 (1998) for IP v4. It is designed to be run to a single autonomous system. Each OSPF router maintains an identical database describing the autonomous system's topology. From this database, a routing table is calculated by constructing a shortest path tree. OSPF recalculates routes quickly during topological changes, using a minimum of routing protocol traffic.

\section{SIMULATION PARAMETERS}

Table 1. Simulation Parameters

\begin{tabular}{|l|l|}
\hline Parameter Name & Parameter Value \\
\hline Terrain Size & $1500 \mathrm{~m} \times 1500 \mathrm{~m}$ \\
\hline Channel Frequency & $2.4 \mathrm{GHz}$ \\
\hline Number of Nodes & 100 \\
\hline Physical Layer & IEEE $802.11 \mathrm{~b}$ \\
\hline Network Layer & IP v4 \\
\hline Mobility Model & Random Waypoint \\
\hline Simulation Time & $30 \mathrm{~s}$ \\
\hline No. Of Packets Sent & 24 per CBR \\
\hline $\begin{array}{l}\text { Minimum/Maximum } \\
\text { Speed }\end{array}$ & $0,5,10,15,20 \mathrm{~m} / \mathrm{s}$ \\
\hline Pause Time & $0 \mathrm{~s}$ \\
\hline
\end{tabular}

The above mentioned simulation parameters are used to compare the ad hoc routing protocols. 5 CBR (Constant Bit Rate) applications between 5 different pairs of nodes are used. The performance parameters i.e. messages received, end to end delay, throughput and jitter are the average of the performance parameters between these 5 pairs.

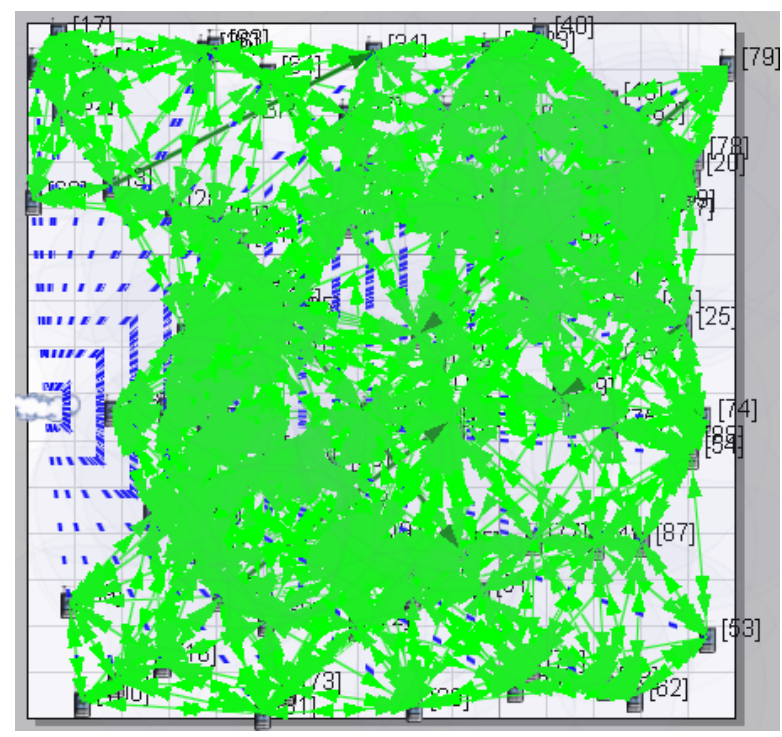

Fig. 2: A simulation canvas running on QualNet 6.1 using MYLAR1 with 100 nodes and 5 CBR 


\section{RESULTS}

\subsection{Total Unicast Messages Received}

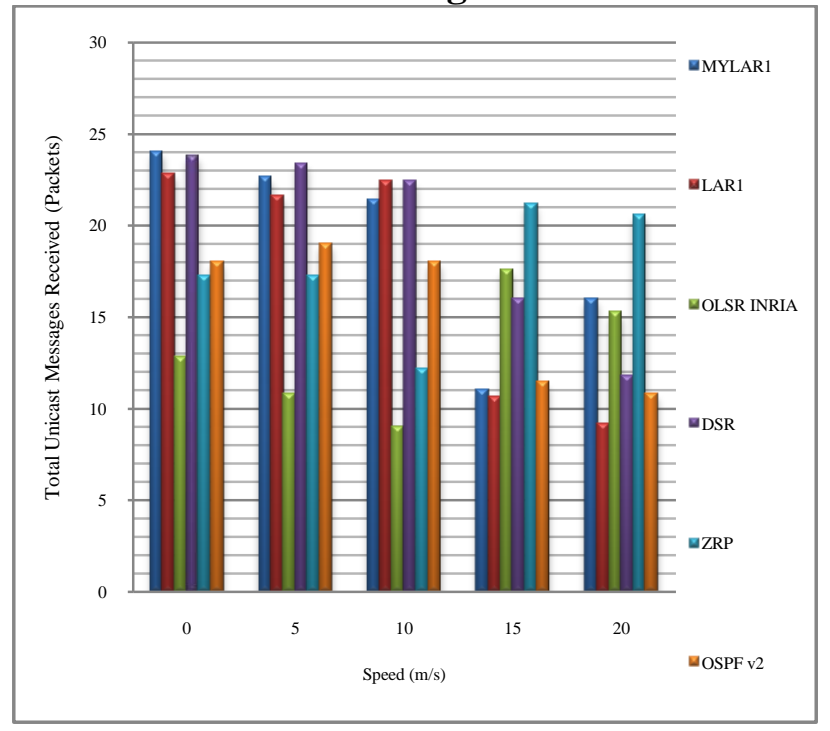

Fig. 3: Total Unicast Messages Received

Total unicast messages received is the total number of unicast messages received (messages received without error) from the beginning of simulation up to the time specified in the timestamp column by all unicast session receivers. From the scenario we see that 24 message packets were send from sender to receiver. From Fig. 3 we observe that MYLAR1 performs significantly well up to $10 \mathrm{~m} / \mathrm{s}$ but above this speed the performance significantly degrades. It is to be noted that up to $10 \mathrm{~m} / \mathrm{s}$ DSR performs the best but above $10 \mathrm{~m} / \mathrm{s}$ its performance degrades. At speed above $10 \mathrm{~m} / \mathrm{s}$ ZRP excels.

\subsection{Average Unicast End to End Delay (s)}

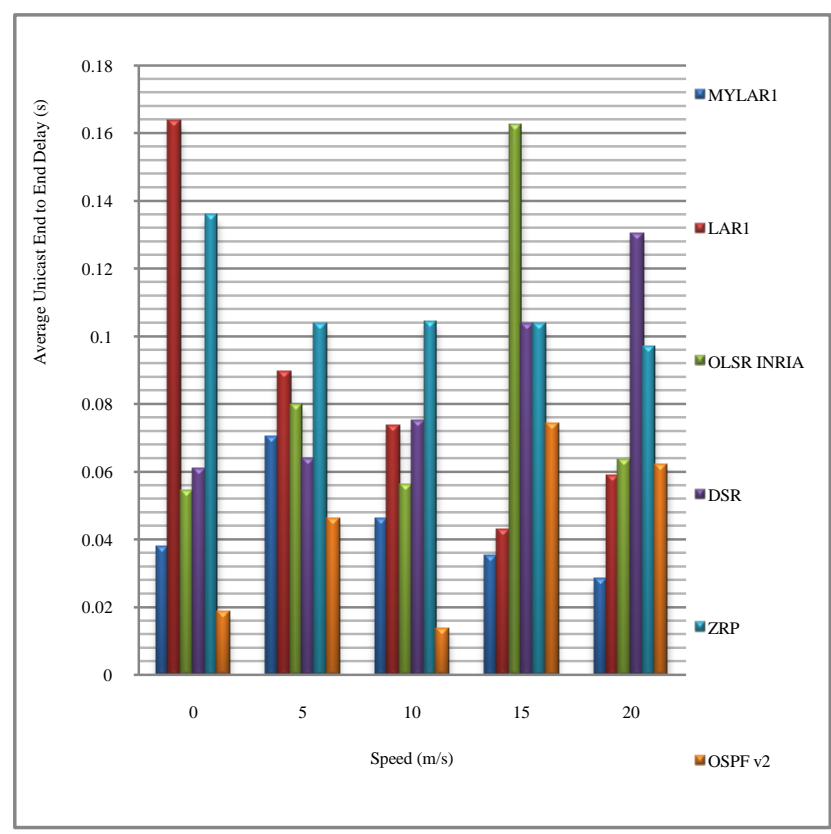

Fig. 4: Average Unicast End to End Delay (s)

Average unicast end to end delay is the overall average unicast message delay from beginning of simulation up to the time specified in the timestamp column. Fig. 4 shows that MYLAR1 performs far better than LAR1 and best above 10 $\mathrm{m} / \mathrm{s}$.

\subsection{Unicast Received Throughput (bits/s)}

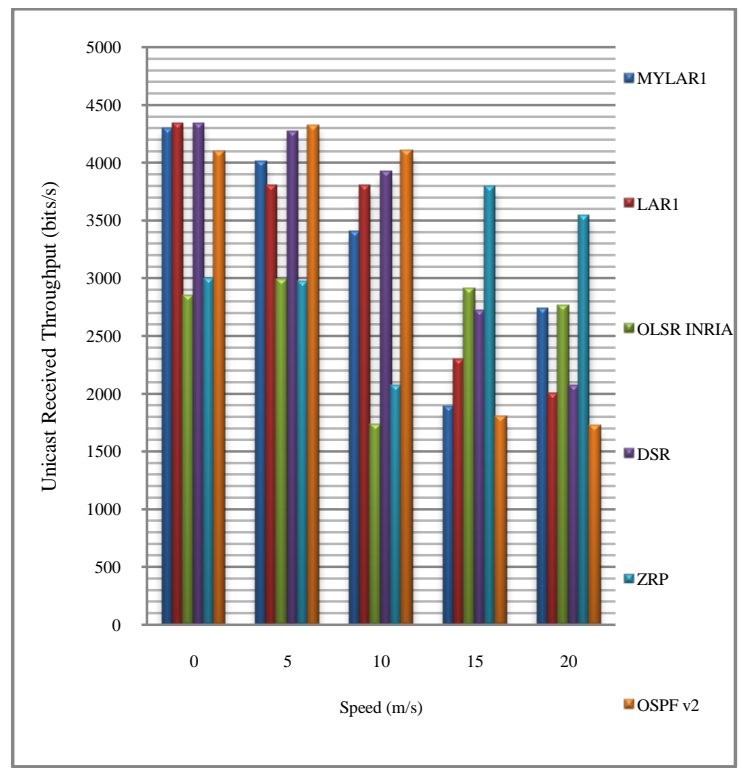

Fig. 5: Unicast Received Throughput (bits/s)

Unicast received throughput is the rate of successful delivery of packets at the receiver. MYLAR1 performs fairly well but at speed greater than $10 \mathrm{~m} / \mathrm{s}$ ZRP outperforms other routing protocols by a significant margin.

\subsection{Average Unicast Jitter (s)}

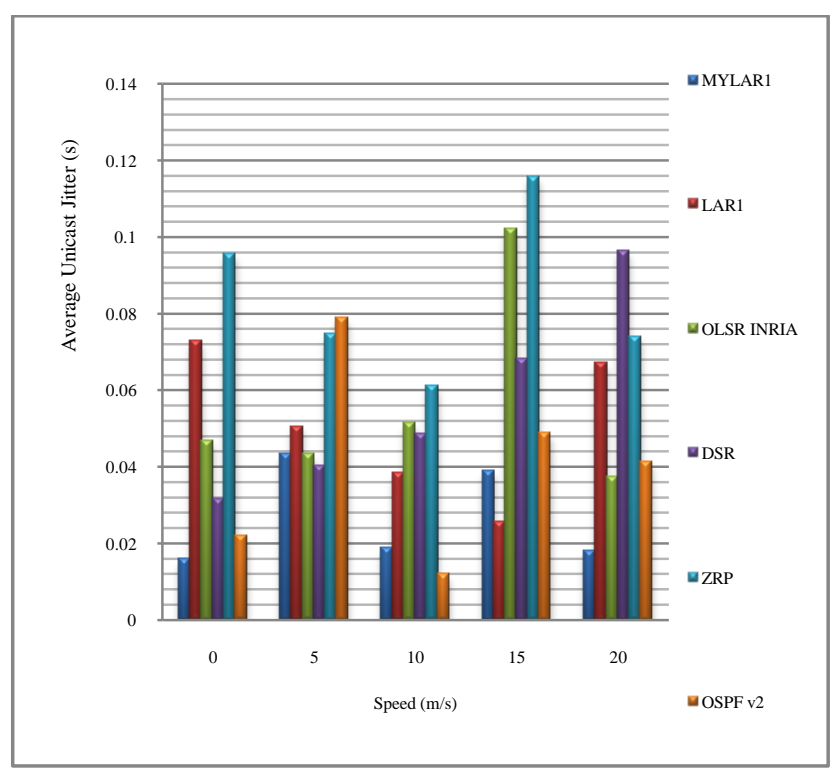

Fig. 6 Average Unicast Jitter (s)

Average unicast jitter is the average difference between end to end delays of successive packets. MYLAR1 clearly outperforms all other routing protocols.

\section{CONCLUSION}

The paper verifies the previous work done that the proposed routing protocol (MYLAR1) is well suited for MANET (Mobile Ad hoc Network) rather than VANET (Vehicular Area Network) because the performance of MYLAR1 degrades with the increasing speed of the nodes in the network. The comparison and analysis of ad hoc routing protocol using QualNet 6.1 Network Simulator shows that the 
proposed modified LAR1 routing protocol i.e. MYLAR1 is a very stable and consistent routing protocol and overall (i.e. taking all the four performance parameters discussed) performs better than LAR1, OLSR INRIA, DSR, ZRP and OSPF v2. The future scope of this work will be to further improve the performance of MYLAR1 by making it more efficient by further reduction of the header size of route request packets and searching for alternative techniques to make MYLAR1 better in all aspects than other routing protocols.

\section{REFERENCES}

[1] S. Corson and J. Macker, "Mobile Ad hoc Networking (MANET): Routing Protocol Performance Issues and Evaluation Considerations", RFC 2501, Jan 1999.

[2] X. Hong, K. Xu and M. Gerla, "Scalable Routing Protocols for Mobile Ad hoc Networks", IEEE Network, 2002, pp. 11-21.

[3] M. Mauve and Jorg Widmer, "A Survey on Position Based Routing in Mobile Ad hoc Networks", IEEE Network, 2001, pp. 30-39.

[4] R. Paulus, A. Jasper and S. Rathore, "Improvement of LAR1 Routing Protocol", ICRTC-2015, Elsevier Computer Procedia, 2015, in press.

[5] Y.B. Co and N.H. Vaidya, "Location Aided Routing (LAR) in Mobile Ad hoc Networks", ACM/IEEE International Conference on Mobile Computing and Networking (MobiCom'98), 1998, pp. 66-75.
[6] S.M. Senouci and T.M. Rasheed, "Modified Location Aided Routing Protocols for Mobile Ad hoc Networks", France Telecom R\&D, 2005.

[7] V.N. Sastry and P. Supraja, "Location Based Associativity Routing for MANET”, IEEE, 2005, pp. 49-8.

[8] F.D. Rango, A. Iera, A. Molinaro, S. Marano, "Multi Step Increase in the Forwarding Zone for LAR Protocols in Ad hoc Networks", IEEE, 2003, pp. 186190.

[9] Rajeev Paulus, Tanbeerbedi, Reema Garg and Ashish, "Performance Comparison of AODV, DSR and LAR1 in Mobile Ad-hoc Network based on Simulation Time", IOSR Journal of Electronics and Communication Engineering (IOSR-JECE) e-ISSN: 2278-2834, pISSN: 2278-8735. Volume 7, Issue 4 (Sep. - Oct. 2013), pp. 32-36.

[10] J. Moy, "OSPF Version 2", The Internet Society, OSPFv2, 1998.

[11] S.V. Singh, R. Paulus, A.K. Jaiswal, A. Kumar, "To Evaluate the Performance of IEEE 802.16 Routing Protocols using QualNet 6.1 Simulator", IJCA, Vol. 92, 2014, pp.

[12] QualNet 6.1 User Guide. 